with longer follow-up and are likely to be largely due to changes in behaviour caused by preclinical dementia.

\section{OP51 PROJECTING THE INCIDENCE AND PREVALENCE OF POST-STROKE COGNITIVE IMPAIRMENT AND DEMENTIA IN THE IRISH POPULATION AGED 40+ YEARS FROM 2015-2025}

${ }^{1}$ E Sexton*, ${ }^{2} \mathrm{NA}$ Donnelly, ${ }^{1} \mathrm{~N}$ Merriman, ${ }^{3} \mathrm{M}$ Guzman-Castillo, ${ }^{3} \mathrm{P}$ Bandosz, ${ }^{2} \mathrm{MA}$ Wren, ${ }^{1} \mathrm{~A}$ Hickey, ${ }^{3} \mathrm{M}$ O'Flaherty, ${ }^{1} \mathrm{~K}$ Bennett. ${ }^{1}$ Division of Population Health, RCSI, Dublin 2, Ireland; ' Social Research, ESRI, Dublin 2, Ireland; ' ${ }^{3}$ Dept of Public Health and Policy, University of Liverpool, Liverpool, UK

10.1136/jech-2019-SSMabstracts.52

Background Post-stroke cognitive impairment (PSCI) is a common consequence of stroke, leading to reduced quality of life and increased care needs. However, rehabilitation services for this condition in Ireland are very limited. The aim was to apply estimates of PSCI incidence to the Irish population and project the number with PSCI in the population in 2025.

Methods We developed a deterministic Markov model to estimate future incidence of PSCI in the population aged 40-89 years living in Ireland up to 2025. Population data, estimates and projections to 2025 were obtained from the Irish Central Statistics Office. Data from the Irish Longitudinal Study on Ageing were used to estimate age and sex specific stroke prevalence in 2014. Age and sex specific stroke incidence was estimated using 2015 public hospital discharge data $(n=6,155)$. Transition probabilities across six health states defined by cognitive impairment, physical disability, dementia and death were estimated using data from stroke survivors in the English Longitudinal Study on Ageing $(n=490)$ (2002-2011). Published data from the South London Stroke Register were used to estimate annual stroke recurrence.

Results The Irish population aged 40-89 years in 2015-2025 $(\mathrm{n}=2.7 \mathrm{~m})$ is projected to have a cumulative incidence of stroke of approximately $2.3 \%$ by $2025(n=63,100)$. Of these incident strokes, approximately $22.5 \%$ are estimated to have died due to stroke $(n=14,200)$, and $23.8 \%$ to have died of another cause $(n=15,000)$ by 2025 . Of the survivors in 2025 $(n=30600)$, approximately $50.9 \%$ are predicted to have cognitive impairment without dementia $(n=15500)$, and $19.4 \%$ to have dementia $(n=5900)$. The total number of stroke survivors is projected to increase from 26700 in 2015 to 41400 in 2025 , equivalent to a $55 \%$ increase in numbers, and the number with post-stroke dementia is projected to more than double from 3900 in 2015 to 8700 in 2025 .

Discussion In 2025, over two thirds of Irish people who have survived a stroke in the preceding 10 years will have cognitive impairment. The number of people with post-stroke dementia is set to double between 2015 and 2025. The model is limited by its deterministic nature, and the assumption that agespecific disease incidence will remain stable. The model will be further developed to include a probabilistic sensitivity analysis, to model alternative scenarios for trends in disease incidence, and to extend the projections to 2035. The model will also be used in an economic evaluation of alternative strategies for stroke management, including cognitive rehabilitation.

\section{OP52 DEMENTIA PROGRESSION IN THE AGEING POPULATION: A COMPUTER SIMULATION ACCOUNTING FOR INDIVIDUAL COGNITIVE AND FUNCTIONAL DECLINE VARIABILITY}

${ }^{1}$ DC Evenden*, ${ }^{1} \mathrm{BM}$ Walsh, ${ }^{2} \mathrm{SC}$ Brailsford, ${ }^{3} \mathrm{PJ}$ Roderick. 'SChool of Health Sciences, University of Southampton, Southampton, UK; ${ }^{2}$ Southampton Business School, University of Southampton, Southampton, UK; ${ }^{3}$ Primary Care and Population Sciences, University of Southamopton, Southampton, UK

10.1136/jech-2019-SSMabstracts.53

Aim To improve planning for the growing older population and the complex care needs of people with dementia, it is important to recognise the variability of individual cognitive and functional decline and associated care costs. However, many studies exploring care demand simplify varied patient trajectories by partitioning dementia patients into severity categories, therefore failing to capture heterogeneity. Our aim was to develop a computer simulation that models dementia progression longitudinally, driven by population-level dementia onset, mortality, and ageing and including individual variability.

Methods Each modelled age group contains two stocks and three flows using the System Dynamics methodology. A cognitively normal (CN) stock is connected via an incident flow to a stock representing people with dementia (PWD). The 65 to 105 year age range is modelled using eight contiguous 5 -year age groups. Ageing is implemented by transferring $\mathrm{CN}$ and PWD survivors from each stock to the next oldest age group at 5 -year intervals. Mortality flows complete the structure for each age group. Published sources provide flow rates. Agentbased methods are used to model individual attributes and outcomes for dementia cases. Progression is modelled by defining a progression rate type for each agent and deriving individual severity progression coefficients. These are based on fixed and random effects regression models, sampled from probability distributions.

Results Mean progression rates are consistent with published studies. By including individualised random effects, the model demonstrates a complex relationship between decline, severity and service use. Rapid decline leads to higher annual care costs with higher mortality rates. Slower decline leads to lower annual care costs over a longer time period. By incorporating these different trajectories within the model, dementia, care costs, and QALYs can be partitioned by fast, intermediate, and slow progression types to more fully support targeted recommendations for planning service delivery.

Discussion Our computer simulation model shows that accounting for the considerable variability in dementia progression rates as well as severity categories provides more accurate representation of the variation in patient trajectories and outcomes. This modelling method hybridises population-level epidemiology and individual-level pathology, allowing future 
simulation scenarios to explore the relative impact of different intervention approaches across the dementia population.

\section{OP53 LONELINESS, SOCIAL ISOLATION AND ALL-CAUSE MORTALITY IN THE OVER 50S IN IRELAND: FINDINGS FROM THE IRISH LONGITUDINAL STUDY ON AGEING (TILDA)}

${ }^{1} \mathrm{M}$ Ward ${ }^{*},{ }^{1,2} \mathrm{~A}$ Nolan, ${ }^{1}$ RA Kenny. ${ }^{1}$ The Irish Longitudinal Study on Ageing (TILDA), School of Medicine, Trinity College Dublin, Dublin, Ireland; ${ }^{2}$ Economic and Social Research Institute, Dublin, Ireland

\subsection{6/jech-2019-SSMabstracts.54}

Background Interest in loneliness and social isolation as risk factors for premature mortality has recently gained increased attention in both the research literature and public discourses. This has resulted in the established of loneliness taskforces in both Ireland and the United Kingdom. Both loneliness and social isolation have previously been linked to a host of adverse health outcomes, including cardiovascular disease, depression, reduced immune and cognitive functioning, and mortality. Loneliness is most often conceptualised as the subjective assessment of an individual's social relationships while social isolation is the objective quantitative measure of social contacts. Loneliness and social isolation that have been shown to be distinct yet synergistic constructs.

This study examined the association between loneliness, social isolation, and all-cause and cardiovascular mortality in Ireland and also tested the hypothesis that loneliness provides a mechanistic pathway that explains the association between social network size and mortality. This is the first study in Ireland to use linked survey-health assessment-mortality data, combining rich individual-level data from the Irish Longitudinal Study on Ageing (TILDA) with official death certificate data provided by the official mortality register.

Methods The sample included 6,800 participants, including 654 decedents (with 199 deaths due to cardiovascular disease) who had participated in the first round of TILDA. Underlying and contributory causes of death were coded using the International Classification of Diseases, Injuries and Causes of Death (ICD-10). Loneliness was measured using the five-item University of California at Los Angeles (UCLA) Loneliness Scale. Social isolation was measured using the Berkman-Syme Social Network Index. Cox proportional hazards ratios were estimated to assess the association between loneliness, social isolation and mortality while controlling for demographic and socio-economic characteristics, health status, and related behaviours. SEM path analysis was used to identify mechanistic pathways.

Results The average age of death was 78 years. After controlling for other factors, the most lonely (HR 1.39 95\% CI: $1.08-1.78, \mathrm{p}<0.01$ ) and most isolated (HR 1.62 95\% CI: $1.21-2.17, \mathrm{p}<0.001)$ had an increased all-cause mortality risk. A similar association was observed between loneliness and cardiovascular deaths (HR 1.68 95\% CI: 1.06-2.66, $\mathrm{p}<0.05$ ). The association between social isolation and mortality was partially mediated by loneliness.

Conclusion These findings add to the growing body of evidence that loneliness and social isolation are associated with premature mortality. They also shed new light on potential mechanistic pathways that partially explain these associations.

\section{OP54 DESCRIBING CHANGE IN MUSCULOSKELETAL AGING: A COMPARISON OF TECHNIQUES USING DATA FROM THE HEALTH, AGING AND BODY COMPOSITION STUDY}

LD Westbury*, HE Syddall, EM Dennison, C Cooper. MRC Lifecourse Epidemiology Unit, University of Southampton, Southampton, UK

\subsection{6/jech-2019-SSMabstracts.55}

Background Preventive strategies for musculoskeletal disorders require a better understanding of age-related changes in muscle strength, physical function and body composition (including bone). Many publications use simple change measures from observations at two time-points which do not account for measurement error and assumes change is linear. Sophisticated techniques for analysing change are available but are rarely implemented in this field.

Methods Changes in grip strength, walking speed, lean mass and hip bone mineral density (BMD) were explored among 3075 men and women from the Health, Aging and Body Composition Study; each measure was assessed at least 5 times during a median 9 year follow-up period. The following techniques were implemented: linear mixed effects models (LMEM) (applied to raw data and age-specific z-scores from generalised additive models for location, scale and shape); growth mixture models (GMM); and latent class trajectory models (LCTM). LMEM use random effects to capture interindividual variation in level and change around a populationaverage trajectory; GMM extend LMEM by identifying clusters of individuals with similar trajectories and deriving cluster-specific average trajectories; and LCTM are simplified GMM with no random effects, assuming all individuals in a cluster have the same trajectory.

Results Mean (SD) age at baseline was 74.1 (2.9) years. Mean annual percentage declines for walking speed and grip strength were $2.1 \%$ and $1.5 \%$ respectively; declines were smaller for hip BMD (0.6\%) and lean mass (0.5\%). Trajectories from LMEM (applied to raw data) for grip strength, walking speed and hip BMD were quadratic in relation to age such that declines accelerated with advancing age; decline in lean mass was linear. Random slopes from LMEM applied to Z-scores were weakly correlated with baseline levels for all characteristics $(-0.36<\mathrm{r}<0.17)$, resulting in person-specific measures of change that were broadly independent of level. All GMM contained a group comprising at least $80 \%$ of the sex-specific sample with sparse numbers of participants in other groups, suggesting that a LMEM with a single population average trajectory describes most of the change in the sample. LCTM derived subgroups with much larger differences in levels of the characteristics rather than in rates of loss.

Conclusion LMEM enable a more comprehensive analysis of change compared to methods using data from only two timepoints. However, inter-individual differences in rates of change regarding musculoskeletal parameters in this age group and duration of follow-up may be too small to be identified using more complex techniques such as GMM or LCTM. 